Pacific Journal of Mathematics

AN INTRINSIC CONSTRUCTION OF FEFFERMAN'S CR 


\title{
AN INTRINSIC CONSTRUCTION OF FEFFERMAN'S CR METRIC
}

\author{
FrANK A. FARris
}

\begin{abstract}
We construct a conformal class of Lorentz metrics naturally associated with an abstract definite $C R$ structure. If the CR structure is that of a pseudoconvex boundary in $C^{n}$ we prove that the intrinsically constructed metric is the same as that discovered by Fefferman using a solution to a complex Monge-Ampère equation. The construction presented here relies on formal solutions of a linear equation, $d \zeta=0$, and provides a relatively simple procedure for computing the metric.
\end{abstract}

I. Introduction. It is known that two strictly pseudoconvex domains of holomorphy in $\mathbf{C}^{n+1}$ are biholomorphically equivalent if and only if the CR structures of their boundaries coincide (see [F1] and [CM]), but current methods for distinguishing among these structures are computationally difficult. Fefferman, in [F2], simplified the situation by constructing a conformal class of Lorentz metrics on a circle bundle over the boundary of a strictly pseudoconvex domain which is invariant under biholomorphism. This was done using a formal approximation to a solution of a complex Monge-Ampère equation near the boundary. Burns et al. [BDS] used the work of Chern and Moser [CM] to show that knowledge of Fefferman's conformal class is enough to discover all the biholomorphic invariants of a boundary.

In this paper we construct a conformal class of Lorentz metrics associated with an abstract definite $\mathrm{CR}$ structure. In the case where the CR structure is that of a hypersurface in $\mathbf{C}^{n+1}$ the metric is shown to coincide with Fefferman's. Since the construction defines the metric in terms of formal solutions to a differential equation of the form $d \zeta=0$ (simpler than the complex Monge-Ampère equation) it should provide a conceptual and computational tool useful in the future study of CR geometry.

The following notations are used:

If $w=w^{1} \wedge \cdots \wedge w^{k}$ is a $k$-form on a vector space $V$ and $w_{1}, \ldots, w_{k}$ are vectors in $V$ then

$$
w\left(W_{1} \wedge \cdots \wedge W_{k}\right)=\operatorname{det}\left(w^{i}\left(W_{j}\right)\right) .
$$


Interior multiplication is defined by

$$
(X\lrcorner w)\left(W_{2} \wedge \cdots \wedge W_{k}\right)=w\left(X \wedge W_{2} \wedge \cdots \wedge W_{k}\right) .
$$

If $w^{1}$ and $w^{2}$ are 1-forms on $V$ we use the symmetric product

$$
w^{1} \cdot w^{2}=w^{1} \otimes w^{2}+w^{2} \otimes w^{1} .
$$

$\mathbf{C}^{*}$ is the multiplicative group $\mathbf{C} \backslash\{0\}$, and if $B$ is a bundle over $M$, $C^{\infty}(M, B)$ is its set of smooth sections.

The author wishes to thank Richard Melrose for his patient encouragement and Jack Lee, who helped considerably in simplifying the arguments of $\S \mathrm{V}$.

II. Abstract CR Structures. Here we tell what is meant by an integrable, definite $\mathrm{CR}$ structure and show how such a structure arises on the boundary of a smoothly bounded strictly pseudoconvex domain in $\mathbf{C}^{n+1}$. It is observed that the CR structure induced on such a boundary near a point $p$ is invariant under a holomorphic change of coordinates near $p$, so that features of the $\mathrm{CR}$ structure are biholomorphic invariants of the domain.

A $C R$ structure on a smooth manifold $M$ of real dimension $2 n+1$ consists of

(i) a subbundle $H M$ of $T M$ of codimension one, and

(ii) a complex structure map $J: H_{P} M \rightarrow H_{P} M$, smooth in $p$, with $J^{2}=-I$.

$J$ induces a splitting of the complexified bundle $H^{\mathrm{C}} M$ into the eigenspaces of $J$ with eigenvalues $i$ and $-i$, called $H^{1,0} M$ and $H^{0,1} M$ respectively.

A CR structure on $M$ is said to be integrable if $H^{1,0} M$ is a subalgebra of $T^{\mathrm{C}} M$ under the Lie bracket, that is

$$
[V, W] \in C^{\infty}\left(M, H^{1,0} M\right) \text { whenever } V, W \in C^{\infty}\left(M, H^{1,0} M\right) .
$$

Dual to $H M$ is a real line bundle $\tilde{E} \subset T^{*} M$ whose sections are forms $\theta$ with $H M \subset \operatorname{ker} \theta$. We say a CR structure is definite at $p$ if, whenever $\theta$ is a nonzero section of $\tilde{E}$ near $p, d \theta(Z \wedge J \bar{Z})$ is nonzero and of fixed sign for any nonzero $Z \in H_{p}^{1,0} M$. This enables us to identify a "positive" half-line bundle $E \subset \tilde{E}$ so that $d \theta(Z \wedge J \bar{Z})>0$ whenever $\theta$ is a section of $E$ near $p$ and $Z$ is non-zero in $H_{p}^{1,0} M$. Then the Levi form

$$
l(X, Y)=d \theta(X \wedge J Y)
$$

is positive-definite on $H M$ and well-defined up to a positive multiple whatever section of $E$ is used.

Note that the orientation given by $\theta \wedge(d \theta)^{n}$ is well-defined if $\theta$ is a section of $E$. 
An abstract CR structure on $M$ allows a decomposition by type of the exterior algebra of $M$ in which, unlike the analogous decomposition for complex manifolds, the type of certain forms is not unique; for instance, any section of $E$ will be of both types $(1,0)$ and $(0,1)$. This will not be pursued in general; the required definitions are

$$
\Lambda^{1,0} M=\left\{\text { complex } 1 \text {-forms } v \text { with } v(\bar{Z})=0 \text { for } \bar{Z} \in H^{0,1} M\right\},
$$

and

$$
\left.\Lambda^{n+1,0} M=\{\text { complex } n+1 \text {-forms } \zeta \text { with } \bar{Z}\lrcorner \zeta=0 \text { for } \bar{Z} \in H^{0,1} M\right\} .
$$

Deleting the zero section of $\Lambda^{n+1,0} M$ we obtain $K$, the canonical bundle of $M$. This $\mathrm{C}^{*}$-bundle is central to the spirit of our approach to $C R$ geometry.

For a motivating context in which these structures arise suppose $M$ is a smooth hypersurface in $\mathbf{C}^{n+1}$. At a point $p$ of $M$ let

$$
H_{p} M=T_{p} M \cap J\left(T_{p} M\right)
$$

where $J$ is the complex structure map of $\mathrm{C}^{n+1}$. Since $J$ restricts to $H_{p} M$ this induces a CR structure on $M$. Both $T^{\mathrm{C}} M$ and the vector fields of type $(1,0)$ in $\mathbf{C}^{n+1}$ form subalgebras of $T^{\mathrm{C}} \mathbf{C}^{n+1}$ and so $H^{1,0}$, belonging to each, is a subalgebra of $T^{\mathrm{C}} M$. Thus the $C R$ structures on hypersurfaces in $C^{n+1}$ are all integrable. Also because of the coordinate-free definition of the induced CR structure, we see that any local holomorphic coordinates near $p$ induce the same CR structure on $M$ near $p$.

The induced CR structure on $M$ is definite at a point $p$ whenever one side of $M$ is strictly pseudoconvex near $p$, as follows: Let $u$ be a smooth defining function for $M$ near $p$ (that is, $d u \neq 0$ along $M$ and $M$ near $p$ is the set where $u$ is zero), and let

$$
\theta=\frac{i \bar{\partial} u-i \partial u}{2}
$$

since $\theta=\bar{\theta}, \theta$ is real, and if $X$ and $J X$ are both tangent to $M$

$$
\theta(X)=\theta\left(\frac{X-i J X}{2}+\frac{X+i J X}{2}\right)=-d u(J X)=0,
$$

so $\theta$ is a section of $\tilde{E}$. The side of $M$ where $u$ is negative is strictly pseudoconvex if and only if $d \theta(Z \wedge J \bar{Z})=\partial \bar{\partial} u(Z \wedge \bar{Z})$ is positive so that $\theta$ is in fact a section of $E$. If $M$ were definite and the form $\theta$ of (2.4) were in the other half of $\tilde{E}$ then the other side of $M$ would be strictly pseudoconvex.

Observe that if $M$ is a smooth hypersurface in $\mathbf{C}^{n+1}$ the coordinate volume form $d z=d z^{1} \wedge d z^{2} \wedge \cdots \wedge d z^{n+1}$ restricts to $M$ to give a 
closed section of $K$. There are various results concerning the natural question of whether an abstract CR structure on $M$ always arises from an embedding of $M$ into $\mathbf{C}^{n+1}$ (see [B], [K], $[\mathbf{J}]$ ). Perhaps alternative proofs and additional results can be obtained by first investigating the existence of a closed section of $K$.

III. First-order CR geometry. In this section we show that a plane tangent to any closed section of $K$, when projected to $C=K / \mathbf{R}^{+}$, is tangent to a member of a certain one-parameter family of cones. Analytically speaking, given the differential of a closed section of $K$, a Lorentz metric on $C$ is constructed and shown to belong to a small class of metrics whose light cones make up the one-parameter family mentioned above. The identification of the conformal class of Fefferman's metrics within the family is accomplished in later sections.

Although the Levi metric of $\S I I$ is well-defined only on $H M$, there is a reasonable way to extend it to $T M$ given the values of $\theta$ and $d \theta$ at a point, that is, given the 1-jet of a section of $E$.

Proposition 3.1. Given $\theta$ and $d \theta$ at a point $p$, there is a unique $T \in T_{p} M$ such that $\theta(T)=1$ and $\left.T\right\lrcorner d \theta=0$.

The proof follows simply from the nondegeneracy of $\left.d \theta\right|_{H}$.

Such a $T$ defines a projection $\pi_{T}: T M \rightarrow H M$ with $\pi_{T}(T)=0$. A natural extension of the Levi metric $l$ to $T M$ is then $h_{\theta}=\pi_{T}^{*}(l)$. Note that it is degenerate and that the metrics arising in this way are in one-to-one correspondence with $H_{P} M$.

To gain intuition about the following construction recall the restriction of a Lorentz metric to a hyperplane tangent to its light cone is degenerate in one direction and positive in others.

This family of degenerate metrics arises in our construction because of a natural way to associate a section $\theta_{\zeta}$ of $E$ to a section $\zeta$ of $K$. $\theta_{\zeta}$ can be thought of as a quantity describing the modulus of $\zeta$. One ingredient in the construction is the metric on $T_{p} M$ obtained from the 1-jet of this modulus.

Proposition 3.2. If $\zeta \in C^{\infty}(M, K)$ there is a unique $\theta_{\zeta} \in C^{\infty}(M, E)$ such that whenever $\theta_{\zeta}(T)=1$

$$
\left.\left.c_{n} \theta_{\zeta} \wedge(T\lrcorner \zeta\right) \wedge(T\lrcorner \bar{\zeta}\right)=\theta_{\zeta} \wedge\left(d \theta_{\zeta}\right)^{n}
$$


where $c_{n}=n ! i^{n}(-1)^{\sigma(n-1)}$ and $\sigma(n-1)=(n-1) n / 2$. Furthermore if $\eta=f \zeta$ with $f$ non-zero then $\theta_{\eta}=|f|^{2 /(n+2)} \theta_{\zeta}$.

Proof. $\theta_{\zeta}$ is found by starting with any section $\theta$ of $E$ and modifying it to satisfy (3.1). Note that both sides are real $2 n+1$-forms.

Form a real $2 n+1$-vector by wedging together the members of the frame $T, Z_{\alpha}, Z_{\bar{\alpha}}$ with $\left.\theta(T)=1, T\right\lrcorner d \theta=0, l\left(Z_{\alpha}, Z_{\bar{\beta}}\right)=0$ unless $\alpha=\beta$, and $l\left(Z_{\alpha}, Z_{\bar{\alpha}}\right)=1$ where $l$ is the Levi form for $\theta$. Computation shows $V=(i)^{n}(-1)^{\sigma(n-1)} T \wedge{ }_{\alpha} Z_{\alpha} \wedge{ }_{\alpha} Z_{\bar{\alpha}}$ is real. Since $\left.c_{n} \theta \wedge(T\lrcorner \zeta\right) \wedge$ $(T\lrcorner \bar{\zeta})(V)=n ! \zeta\left(T \wedge Z_{\alpha}\right) \bar{\zeta}\left(T \wedge Z_{\bar{\alpha}}\right)$ while $\theta \wedge(d \theta)^{n}(V)=n !$ the first $2 n+1$-form must be a positive multiple of the second. Say

$$
\left.\left.c_{n} \theta \wedge(T\lrcorner \zeta\right) \wedge(T\lrcorner \bar{\zeta}\right)=\lambda \theta \wedge(d \theta)^{n} \quad \text { where } \lambda>0 .
$$

$\theta_{\zeta}=\lambda^{1 /(n+2)} \theta$ can be seen to satisfy (3.1). The transformation law is similarly verified.

Note that the value of $\theta_{\zeta}$ at $p$ depends only on the value of $\zeta$ at $p$ and that $\zeta$ and $e^{i f \zeta}$ give rise to the same sections of $E$. This last observation makes it plausible to think of $\theta_{\zeta}$ as a quantity representative of the modulus of $\zeta$. Because $\theta_{\zeta}$ is determined through a comparison of volume forms in (3.1) it is called the volume-normalized section of $E$ corresponding to $\zeta$.

The last ingredient in the construction of the metric related to $\zeta$ is the plane tangent to the closed section $\zeta$. The analytic object involved is a function $F$ on $C$ whose level set is the image of the map $\zeta: M \rightarrow C$. The same notation is used for the maps $\zeta: M \rightarrow K$ and $\zeta: M \rightarrow C$. When a distinction is necessary [ $\zeta$ ] will denote the equivalence class of $\zeta$ in the quotient $K / \mathbf{R}^{+} . F$ is defined by

$F\left(\left[e^{l \alpha} \zeta(p)\right]\right)=\alpha$ for $-\pi<\alpha<\pi$ and $p \in M$. The tangent to the $S^{1}$ action on $C\left(\zeta \rightarrow e^{i \alpha} \zeta\right)$ is called $S$. Clearly if $\sigma=d F, \sigma(S)=1$.

Now let $\theta$ denote $\theta_{\zeta}$, the volume-normalized section of $E$ and find $T$ as in Proposition 1. Let $h_{\theta}$ be the extended Levi metric and let $\pi$ : $T_{\zeta} C \rightarrow T_{p} M$ be the natural projection. Then $g_{\zeta}$ is defined by

$$
g_{\zeta}=\pi^{*} h_{\theta}+\frac{2}{n+2} \theta \cdot \sigma .
$$

( $\theta$ and $\pi^{*} \theta$ are identified.)

THEOREM. If $\zeta$ is a closed section of $K$ and $\eta=f \zeta$ is also closed with $f(p)=1$ then

$$
g_{\eta}-g_{\zeta}=c \theta_{\zeta}^{2}
$$


Moreover if $d f=a \theta+a_{\alpha} \theta^{\alpha}$ where $\theta, \theta^{\alpha}, \theta^{\bar{\alpha}}$ is the coframe dual to the frame of Proposition 3.2, then

$$
c=\frac{a_{\alpha} a_{\bar{\alpha}}}{(n+2)^{2}}+i \frac{(a-\bar{a})}{n+2} .
$$

Proof. Both sides of (3.3) will be evaluated on all pairs of vectors in a basis for $T_{\zeta} C$. A convenient basis consists of $S$ together with the frame $T$, $Z_{\alpha}, Z_{\bar{\alpha}}$ of Proposition 3.2 lifted without renaming to the plane tangent to the section $\zeta$.

It is immediate from (3.2) that both sides of (3.3) are zero on the lift of $H$, since both $g_{\eta}$ and $g_{\zeta}$ are essentially the Levi form there. Also $\delta$ is perpendicular to $Z_{\alpha}, Z_{\bar{\alpha}}$, and $S$ in both metrics $g_{\zeta}$ and $g_{\eta}$ so (3.3) is true on these pairs. Similarly $g_{\zeta}(T, S)=g_{\eta}(T, S)=2 /(n+2)$.

It remains to show that (3.3) evaluated on the pairs $\left(T, Z_{\alpha}\right)$ and $\left(T, Z_{\bar{\alpha}}\right)$ gives zero on both sides and that $\left(g_{\eta}-g_{\xi}\right)(T, T)=2 c$.

To verify these differentiate $\theta_{\eta}=|f|^{2 /(n+2)} \theta_{\zeta}$ and find that

$$
T_{\eta}=t T_{1}+t^{\alpha} Z_{\alpha}+t^{\bar{\alpha}} Z_{\bar{\alpha}},
$$

where

$$
t=|f|^{-2 /(n+2)} \quad \text { and } \quad t^{\alpha}=\frac{-i a_{\bar{\alpha}}}{(n+2) \bar{f}|f|^{2 /(n+2)}},
$$

satisfies the conditions of Proposition 3.1 for $\theta_{\eta}$.

Computation shows that since $F_{\eta}=F_{\zeta}-\arg f$

$$
\sigma_{\eta}=\sigma_{\zeta}+\frac{i}{2}\left\{a_{\alpha} \theta^{\alpha}-a_{\bar{\alpha}} \theta^{\bar{\alpha}}+(a-\bar{a}) \theta\right\} \quad \text { at } p \text {. }
$$

Thus the term gained in $\pi^{*} h_{\theta_{\eta}}\left(T, Z_{\alpha}\right)$ cancels that in $\sigma_{\eta} \cdot \theta\left(T, Z_{\alpha}\right)$ in the expression $g_{\eta}\left(T, Z_{\alpha}\right)$.

Finally compute that

$$
g_{\eta}(T, T)=2 \frac{a_{\alpha} a_{\bar{\alpha}}}{(n+2)^{2}}+2 \frac{(a-\bar{a})}{(n+2)}=2 c .
$$

Since $g_{\zeta}(T, T)=0$ and $\theta^{2}(T, T)=2$ this establishes the theorem.

Even at this stage the construction may be useful in investigating the behavior of the chains, which are the light rays of the metric of $\S I V$. The dynamical system for the light rays of $g_{\zeta}$ is easy to compute. If any property of this simple dynamical system could be shown invariant under the addition of a $\theta^{2}$ term to the metric, it would necessarily be a property of the chains. 
IV. Second-order CR geometry. A particular conformal class within the small family of Lorentz metrics from §III is determined using a small part of the 2-jet of any closed section of $K$. Actually there need not be any closed section for the construction to work: to find the Fefferman metric at $p$ the reconstruction from formal power series of a closed 2-jet of section of $K$ over $p$ is sufficient. for simplicity of language, in this section the required 2-jets are referred to as closed sections, although in the most general case such sections may not exist.

Proposition 4.1. If $\zeta$ is a closed section of $K, \theta$ its volume-normalized section of $E$, and $T$ the vector field with $\theta(T)=1, T\lrcorner d \theta=0$, then there is a real function $\alpha_{\zeta} \in C^{\infty}(M)$ such that

$$
\left.\left.\left.\mathscr{L}_{T} \zeta \wedge(T\lrcorner \bar{\zeta}\right)=i \alpha_{\zeta} \theta \wedge(T\lrcorner \zeta\right) \wedge(T\lrcorner \bar{\zeta}\right) .
$$

The mapping $\zeta \mapsto \alpha_{\zeta}$ is a second-order non-linear differential operator.

Proof. Interior multiplication of (3.1) by $T$ gives $\left.\left.c_{n}(T\lrcorner \zeta\right) \wedge(T\lrcorner \bar{\zeta}\right)=$ $(d \theta)^{n}$. Exterior differentiation of this equation gives

$$
\left.\left.\mathscr{L}_{T} \zeta \wedge(T\lrcorner \zeta\right)+(T\lrcorner \zeta\right) \wedge \mathscr{L}_{T} \bar{\zeta}=0 .
$$

The complex conjugate of the first term is equal to the second term, so the left-hand side of (4.1) is a purely imaginary multiple of the positive volume form $\theta \wedge(d \theta)^{n}$. This establishes the existence of the function $\alpha_{\zeta}$ as claimed.

To see that the correspondence $\zeta \mapsto \alpha_{\zeta}$ is the work of a second-order differential operator, observe that $T$ and $T\lrcorner \zeta$ depend on the 1-jet of $\zeta$ so that another differentiation depends on the 2-jet of $\zeta$. Of course, wedging with $T\lrcorner \bar{\zeta}$ eliminates much of the information from the 2-jet of $\zeta ; \alpha_{\zeta}$ involves only a small part of this. Non-linearity follows from the formula $\alpha_{c \zeta}=|c|^{-2 /(n+1)} \alpha_{\zeta}$, when $c$ is constant.

The proof that $\alpha_{\zeta}$ can be used to fix the undetermined $\theta^{2}$ term in the metrics of $\S$ III is facilitated by the choice of a special frame for TM near $p$, similar to the frame of Proposition 3.2, but enjoying special bracket relations identical at $p$ to the celebrated ones on the Heisenberg group.

Proposition 4.2. Given $\theta \in C^{\infty}(M, E)$, near any point of $p$ of $M$ there are vector fields $T, Z_{\alpha}$ such that

(a) $\theta(T)=1, T\lrcorner d \theta=0$,

(b) $\left[Z_{\alpha}, Z_{\bar{\alpha}}\right]=-i T$ at $p$, and

(c) all other bracket relations among the vector fields $T, Z_{\alpha}, Z_{\bar{\alpha}}$ are zero at $p$. 
Proof. (a) is established by taking $T$ as in Proposition 3.1. To find the vector fields $Z_{\alpha}$, start with any vector fields $Z^{\prime}$ matching at $p$ the frame of Proposition 3.2 so that at $p$

$$
d \theta\left(Z_{\alpha} \wedge Z_{\bar{\beta}}\right)=i \delta_{\alpha \bar{\beta}} .
$$

These will be modified by finding $n^{2}$ smooth complex functions $r_{\alpha}^{\beta}$ vanishing at $p$ so that the vector fields

$$
Z_{\alpha}=Z_{\alpha}^{\prime}+r_{\alpha}^{\beta} Z_{\beta}^{\prime}
$$

satisfy the given conditions. Note that the type of $Z_{\alpha}$ is still $(1,0)$ and that the condition $r_{\alpha}^{\beta}(p)=0$ preserves the orthonormality of these vectors with respect to the Levi form at $p$, which in turn guarantees that (b) and (c) hold modulo vectors in $H$.

\section{Writing}

$$
\left[Z_{A}^{\prime}, Z_{B}^{\prime}\right]=c_{A B} T+d_{A B}^{c} Z_{c}^{\prime} \text { and }\left[T, Z_{A}^{\prime}\right]=e_{A} T+f_{A}^{B} Z_{B},
$$

where $A, B$, and $\mathrm{d} C$ take on the values $1,2, \ldots, n, \overline{1}, \overline{2}, \ldots, \bar{n}$, it's easy to see that

$$
c_{A B}= \begin{cases}-i & \text { if } A=\alpha, B=\bar{\alpha} \\ i & \text { if } A=\bar{\alpha}, B=\alpha \\ 0 & \text { otherwise }\end{cases}
$$

and $e_{A}=0$. Thus the $T$ components of the left-hand side of the bracket relations are already correct.

The quantities $d_{A B}^{c}$ and $f_{A}^{B}$ must be elminated by the choice of the functions $r_{\alpha}^{\beta}$. At first it seems that too many terms are to be cancelled by too few choices, but calculation shows that if the equations

$$
\begin{aligned}
& d_{\alpha \bar{\gamma}}^{\beta}=Z_{\bar{\gamma}} r_{\alpha}^{\beta} \\
& d_{\alpha \gamma}^{\beta}=Z_{\gamma} r_{\alpha}^{\beta}-Z_{\alpha} r_{\gamma}^{\beta} \\
& \operatorname{Tr}_{\alpha}^{\beta}=f_{\alpha}^{\beta}
\end{aligned}
$$

are satisfied at $p$, then all the desired bracket relations will hold there. But these are $4 n^{3}+2 n^{2}$ real equations for the $2 n^{2}(2 n+1)$ derivatives of the real and imaginary parts of the functions $r_{\alpha}^{\beta}$ at $p$. Since these may be specified at will the functions $r_{\alpha}^{\beta}$ may be found as claimed. Note how the proof relies on the integrability of the CR structure since this ensures that $d_{\beta \gamma}^{\bar{\alpha}}=d_{\bar{\beta} \bar{\gamma}}^{\alpha}=0$. If these were non-zero too many conditions would be imposed on the functions $r_{\alpha}^{\beta}$.

Now the metric will be constructed. 
THEOREM. Let $g_{\zeta}$ be the metric on $C$ associated with a closed section $\zeta$ of $K$. Let

$$
g=g_{\zeta}+\frac{\alpha_{\zeta}}{n+1} \theta_{\zeta}^{2} .
$$

Then the conformal class of $g$ is independent of $\zeta$.

Proof. Consider changing $\zeta$ to $f \zeta$ where $\partial_{\bar{b}} f=0$ and $f(p)=1$. As before write

$$
d f=a \theta+a_{\alpha} \theta^{\alpha}
$$

where now the forms $\theta, \theta^{\alpha}, \theta^{\bar{\alpha}}$ are dual to the frame $T, Z_{\alpha}, Z_{\bar{\alpha}}$ of Proposition 4.2, which is modified by a constant multiple of modulus 1 , if necessary, so that

$$
\zeta\left(T \wedge_{\alpha} Z_{\alpha}\right)=1 \text { at } p .
$$

Since the transformation law relating $g_{f \zeta}$ to $g_{\zeta}$ is known from §III it remains to find $\alpha_{f \zeta}$ in terms of $\alpha_{\zeta}$ and $f$. This is straightforward using our special frame. Evaluate both sides of the equation defining $\alpha_{f \zeta}$ on the $(2 n+1)$-vector $T \wedge_{\alpha} Z_{\alpha} \wedge_{\bar{\alpha}} Z_{\bar{\alpha}}$ to find

$$
\left.d\left(T_{f \zeta}\right\lrcorner f \zeta\right)\left(T \wedge_{\alpha} Z_{\alpha}\right)=i \alpha_{f \zeta}|f|^{-2 /(n+2)} f \bar{f} .
$$

The left-hand side will be expanded using Lie-brackets. This is simple because the vector fields $T, Z_{\alpha}$ all commute. We find

$$
\begin{aligned}
i \alpha_{f \zeta}|f|^{(n-2) /(n+2)}= & \left.d\left(f|f|^{-2 /(n+2)}\right)(T)+f|f|^{-2 /(n+2)} d(T\lrcorner \zeta\right)\left(T \wedge_{\alpha} Z_{\alpha}\right) \\
& -Z_{\alpha}\left(\frac{i a_{\bar{\alpha}} f}{(n+2) \bar{f}|f|^{2 /(n+1)}}\right)
\end{aligned}
$$

The term involving $Z_{\alpha} a_{\bar{\alpha}}=Z_{\alpha} Z_{\bar{\alpha}} \bar{f}$ can be simplified using the bracket relations:

$$
Z_{\alpha} Z_{\bar{\alpha}} \bar{f}=\left(-i T+Z_{\bar{\alpha}} Z_{\alpha}\right) \bar{f}=-i \bar{a} \quad \text { for each } \alpha .
$$

Computing the other terms and setting $f=1$ after differentiating gives

$$
\alpha_{f \zeta}=-i \frac{(n+1)}{n+2}(a-\bar{a})-\frac{n+1}{(n+2)^{2}} a_{\alpha} a_{\bar{\alpha}}+\alpha_{\zeta}
$$

Combining this with the transformation law for $g_{f \zeta}$ (3.6) shows

$$
g_{f \zeta}+\frac{\alpha_{f \zeta}}{n+1} \theta^{2}=g_{\zeta}+\frac{\alpha_{\zeta}}{n+1} \theta^{2}
$$

so the metric is well-defined at $p$ using either $\zeta$ or $f \zeta$ provided $f(p)=1$. To include more general changes of section observe that changing $\zeta$ to $c \zeta$, 
where $c$ is constant, changes $g$ to $|c|^{2 /(n+2)} g$. Then changing $\zeta$ to $f \zeta$ where $f(p)=c$ can be accomplished by first changing $\zeta$ to $c \zeta$ and then to $(f / c)(c \zeta)$, leaving the conformal class of $g$ invariant.

Since the class of closed 2-jets of sections of $K$ depends only on the CR structure of $M$, and since the construction above assigns the same conformal class of metrics to each of these, this conformal class of metrics on $C$ is seen to be a natural feature of the CR structure of $M$.

Those familiar with Chern's construction of an invariant connection on a principal fiber bundle over a $\mathrm{CR}$ manifold from which the same conformal class of Lorentz metrics can be derived may observe that the construction here involves fewer normalizations and differentiations. The author believes that locating the construction on $K$ rather than $E$ leads to this simplicity.

Finally we summarize the construction. To compute the metric choose $\zeta$ a closed section of $K$ and find $\theta_{\zeta}$ the volume normalized section of $E$. Use $d \theta_{\zeta}$ and $\sigma_{\zeta}$ to compute the metric $g_{\zeta}$ which may differ from the true metric by a term $\theta^{2}$. To fix this term differentiate $\zeta$ again to determine $\alpha_{\zeta}$ and write down the metric as in (4.5).

V. The complex Monge-Ampère equation. Fefferman, in [F2], defines a conformal class of Lorentz metrics on $S^{1} \times \partial \Omega$ where $\Omega$ is a smoothly bounded strictly pseudoconvex domain in $\mathbf{C}^{n+1}$, using a second-order approximation of a solution to the complex Monge-Ampère equation in $\Omega$. Here we show that the conformal class of Lorentz metrics defined in $\S \mathrm{IV}$ is the same as Fefferman's in the case where $M$ is embedded near $p$ as part of $\partial \Omega$. This is done by means of a reformulation of the MongeAmpère equation in terms of volume forms.

The equation studied by Fefferman is

$$
J(u)=(-1)^{n+1} \operatorname{det}\left(\begin{array}{cc}
u & u_{\bar{k}} \\
u_{j} & u_{j \bar{k}}
\end{array}\right)=1
$$

where

$$
u_{J}=\frac{\partial u}{\partial z^{\prime}}, \quad u_{\bar{k}}=\frac{\partial u}{\partial z^{\bar{k}}}, \quad u_{\bar{\jmath}}=\frac{\partial^{2} u}{\partial z^{j} \partial z^{\bar{k}}} .
$$

Cheng and Yau $[\mathbf{C Y}]$ showed the existence and uniqueness of a function $u$ satisfying (5.1) in $\Omega$ and the boundary condition

$$
u=0 \text { on } \partial \Omega
$$

so that $u$ is a special defining function for $\Omega$. This result is not necessary to Fefferman's construction, which uses a second-order approximation along $\partial \Omega$ of $u$ satisfying (5.1) and (5.2). 
Equation (5.1) can be written as a comparison of two volume forms in $\mathrm{C} \times \Omega$, where we take $\left(z^{0}, z^{1}, \ldots, z^{n+1}\right)$ as coordinates. Introduce the real $(1,1)$ form

$$
\phi=i u d z^{0} \wedge d z^{\overline{0}}+i \partial u \wedge d z^{\overline{0}}+i d z^{0} \wedge \bar{\partial} u+i \partial \bar{\partial} u .
$$

Then $\phi^{n+2}$ is calculated in two ways. First, directly from (5.3),

$$
\begin{aligned}
\phi^{n+2}= & i^{n+2}(n+2) u d z^{0} \wedge d z^{\overline{0}} \wedge(\partial \bar{\partial} u)^{n+1} \\
& -i^{n+2}(n+2)(n+1) d z^{0} \wedge d z^{\overline{0}} \wedge \partial u \wedge \bar{\partial} u \wedge(\partial \bar{\partial} u)^{n}
\end{aligned}
$$

But it is also true that if

$$
\begin{aligned}
\eta & =\eta_{j \bar{k}} d z^{j} \wedge d z^{\bar{k}} \text { in } \mathbf{C}^{n+2} \\
\eta^{n+2} & =(-1)^{(n+2)(n+1) / 2}(n+2) ! \operatorname{det}\left(\eta_{j \bar{k}}\right) d z^{0} \wedge d z \wedge d z^{\overline{0}} \wedge d \bar{z}
\end{aligned}
$$

where here, as below, $d z=d z^{1} \wedge \cdots \wedge d z^{n+1}$. Applying this to $\phi$ shows

$$
\phi^{n+2}=(-1)^{(n+2)(n+1) / 2}(n+2) ! i^{n+2} J(u) d z^{0} \wedge d z^{\overline{0}} \wedge d z \wedge d \bar{z} .
$$

Contracting with $\partial / \partial z^{0}$ and $\partial / \partial z^{\overline{0}}$ and cancelling $i(n+2)$ we see that $u$ satisfies (5.1) if and only if

$$
\begin{aligned}
i^{n+1}\left(u(\partial \bar{\partial} u)^{n+1}-(n+1) \partial u\right. & \left.\wedge \bar{\partial} u \wedge(\partial \bar{\partial} u)^{n}\right) \\
= & (-1)^{(n+2)(n+1) / 2}(n+1) ! i^{n+1} d z \wedge d \bar{z}
\end{aligned}
$$

Now assuming that (5.6) holds on $\partial \Omega$ we find that $(i \bar{\partial} u-i \partial u) / 2$ is volume normalized with respect to $d z$. Then taking $\zeta=d z, \alpha_{\zeta}$ is computed, assuming that (5.6) can be differentiated in a certain direction transverse to the boundary; since this direction is found by differentiating $u$ this assumption amounts to requiring that (5.6) holds to second order along $\partial \Omega$. Then the metric constructed in $\S \S I I I$ and IV can be shown to belong to Fefferman's conformal class

Some extrinsic quantities useful in this computation are defined by the following proposition.

Proposition 5.1. For any defining function $u$ there are near $\partial \Omega$ a smooth real function $r$ and a vector field $\xi$ of type $(1,0)$ such that

$$
\partial u(\xi)=1 \text { and } \xi\lrcorner i \partial \bar{\partial} u=i r \bar{\partial} u .
$$

The proof is straightforward using the nondegeneracy of $i \partial \bar{\partial} u$ on $H$.

Note that $r$ may be 0 if $i \partial \bar{\partial} u$ is degenerate.

Proposition 5.2. If $J(u)=1$ on $\partial \Omega$, then $\theta=(i \bar{\partial} u-i \partial u) / 2$ is the volume normalized section of $E$ corresponding to $d z$ and $\partial \Omega$. 
Proof. Contracting (5.6) with $\xi$ and $\bar{\xi}$, setting $u=0$ and ignoring terms which vanish on $T M$ gives

$$
\left.\left.-(i \partial \bar{\partial} u)^{n}=(-1)^{(n+2)(n+1) / 2} n ! i^{n}(\xi\lrcorner \zeta\right) \wedge(\bar{\xi}\lrcorner \bar{\zeta}\right) \quad \text { on } T M .
$$

Set $T=i(\xi-\bar{\xi})$ so that $T$ is as in Proposition 3.1. Then wedge (5.8) with $\theta$ and replace $\xi\lrcorner \zeta$ by $-i T\lrcorner \zeta$ to find

$$
\left.\left.\theta \wedge(d \theta)^{n}=(-1)^{n(n-1) / 2} n ! i^{n} \theta \wedge(T\lrcorner \zeta\right) \wedge(T\lrcorner \bar{\zeta}\right)
$$

which is exactly the volume normalization.

Next the Lie derivative of (5.6) with respect to $\xi$, which is transverse to $\partial \Omega$, is used to compute $\alpha$ in terms of $r$.

Proposition 5.3. If $\zeta=d z, \alpha_{\zeta}=(n+1) r$.

Proof. Since $\alpha$ is defined by a comparison of $2 n+1$-forms on $\partial \Omega$ (5.6) will be contracted with $\xi$ before differentiation. This gives

(5.10) $\left.i^{n+1}(u r-1) \bar{\partial} u \wedge(\partial \bar{\partial} u)^{n}=(-1)^{(n+2)(n+1) / 2} n ! i^{n+1}((\xi\lrcorner d z) \wedge d \bar{z}\right)$.

Lie differentiation with respect to $\xi$ of the left-hand side gives $-i^{n+1} n r \bar{\partial} u$ $\wedge(\partial \bar{\partial} u)^{n}$.

To differentiate the other side introduce $\eta=\xi\lrcorner d z$ so that $\zeta=\partial u \wedge \eta$. Differentiating this formula, multiplying in $\xi$, and wedging with $\bar{\eta}$ gives

$$
(\xi\lrcorner d \eta) \wedge \bar{\zeta}=(-1)^{n}\{r \bar{\partial} u \wedge \eta \wedge \bar{\eta}+d \eta \wedge \bar{\eta}\} .
$$

Therefore the Lie derivative of the right-hand side is

$$
(-1)^{n(n-1) / 2} n ! i^{n+1}\{r \bar{\partial} u \wedge \eta \wedge \bar{\eta}+d \eta \wedge \bar{\eta}\} .
$$

Since $\alpha$ is defined by

$$
d \eta \wedge \bar{\eta}=i \alpha \theta \wedge \eta \wedge \bar{\eta}
$$

equating the two Lie derivatives gives

$$
-n r \theta \wedge(d \theta)^{n}=(-1)^{n(n-1) / 2} n ! i^{n}(r-\alpha)(\theta \wedge \eta \wedge \bar{\eta}) .
$$

Using (5.9) gives $\alpha=(n+1) r$.

The metric defined on $S^{1} \times \partial \Omega$ by Fefferman is

$$
f=\frac{i}{n+2}(\bar{\partial} u-\partial u) \cdot d s+u_{j \bar{k}} d z^{j} \cdot d z^{\bar{k}},
$$

where $s$ is the angular variable on $S^{1}$. To compare this with the metric $g$ consider $\zeta$ as trivializing the circle bundle $C$ over $\partial \Omega$ so that $s$ is the function $F$ of $\S I V$. 
THEOREM. $g=f$. Therefore the conformal class constructed in §§III and IV coincides with that constructed by Fefferman.

Proof. First observe that the metrics agree on the maximal complex tangent space $H$, since each agrees with the Levi form corresponding to $i \partial \bar{\partial} u$ there. Also both $S$ and $T$ are perpendicular to $H$ in each metric. $S$ is null in each and $g(S, T)=2 /(n+2)=f(S, T)$. It remains to show that $g(T, T)=f(T, T)$.

$$
f(T, T)=i \partial \bar{\partial} u(T \wedge J T)=2 r .
$$

On the other hand $g(T, T)=2 \alpha /(n+1)$; but $\alpha=(n+1) r$ so the metric agree on a set of basis vectors and are therefore equal.

\section{REFERENCES}

[B] L. Boutet de Monvel, Integration des équations de Cauchy-Riemann induites formelles, Séminaire Goulaouic-Lions-Schwartz 1974-75: Équations aux derivées partielles linéaires et non linéaires, Exp. No. 9, 14 pp. Centre Math., Ecole Polytech., Paris, 1975.

[BDS] D. Burns, Jr., K. Diederich and S. Shnider, Distinguished curves in pseudoconvex boundaries, Duke Math. J., 44 (1977), 407-431.

[CY] S. Y. Cheng and S. T. Yau, On the existence of a complete Kähler metric on non-compact complex manifolds and the regularity of Fefferman's equation, Comm. Pure Appl. Math., 33 (1980), 507-544.

[CM] S. S. Chern and J. K. Moser, Real hypersurfaces in complex manifolds, Acta Math., 133 (1980), 507-544.

[F1] C. Fefferman, The Bergman kernel and biholomorphic mappings of pseudoconvex domains, Invent. Math., 26 (1974), 1-65.

[F2] _ Monge-Ampère equations, the Bergman kernel, and geometry of pseudoconvex domains, Annals of Math., 193 (1976), 395-416.

[J] H. Jacobowitz and F. Trèves, Nonrealizable CR structures, Invent. Math., 66 (1982), 231-249.

[K] M. Kuranishi, Strongly pseudoconvex CR structures over small balls Part III. An embedding theorem, Annals of Math., 116 (1982), 249-330.

Received November 5, 1984.

University of Santa Clara

Santa Clara, CA 95053 



\section{PACIFIC JOURNAL OF MATHEMATICS EDITORS}

V. S. VARADARAJAN

(Managing Editor)

University of California

Los Angeles, CA 90024

HERBERT CLEMENS

University of Utah

Salt Lake City, UT 84112

R. FINN

Stanford University

Stanford, CA 94305
HERMANN FLASCHKA

University of Arizona

Tucson, AZ 85721

RAMESH A. GANGOLLI

University of Washington

Seattle, WA 98195

VAUGHAN F. R. JONES

University of California

Berkeley, CA 94720

ROBION KIRBY

University of California

Berkeley, CA 94720
C. C. MOORE

University of California

Berkeley, CA 94720

H. SAMELSON

Stanford University

Stanford, CA 94305

HAROLD STARK

University of California, San Diego

La Jolla, CA 92093

\section{ASSOCIATE EDITORS}

R. ARENS

E. F. BECKENBACH

B. H. NEUMANN (1906-1982)
F. WOLF

K. YOSHIDA

\section{SUPPORTING INSTITUTIONS}

UNIVERSITY OF ARIZONA

UNIVERSITY OF BRITISH COLUMBIA

CALIFORNIA INSTITUTE OF TECHNOLOGY

UNIVERSITY OF CALIFORNIA

MONTANA STATE UNIVERSITY

UNIVERSITY OF NEVADA, RENO

NEW MEXICO STATE UNIVERSITY

OREGON STATE UNIVERSITY
UNIVERSITY OF OREGON UNIVERSITY OF SOUTHERN CALIFORNIA

STANFORD UNIVERSITY

UNIVERSITY OF HAWAII

UNIVERSITY OF TOKYO

UNIVERSITY OF UTAH

WASHINGTON STATE UNIVERSITY

UNIVERSITY OF WASHINGTON 


\section{Pacific Journal of Mathematics}

\section{Vol. 123, No. $1 \quad$ March, 1986}

Maria Emilia Alonso García, A note on orderings on algebraic varieties $\ldots \ldots 1$

F. S. De Blasi and Józef Myjak, On continuous approximations for

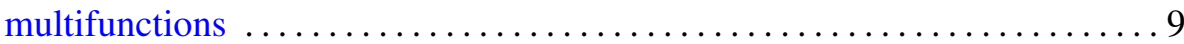

Frank Albert Farris, An intrinsic construction of Fefferman's CR metric . . . 33 Antonio Giambruno, P. Misso and Francisco César Polcino Milies, Derivations with invertible values in rings with involution $\ldots . \ldots . \ldots .47$

Dan Haran and Moshe Jarden, The absolute Galois group of a pseudo real

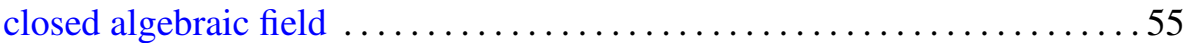

Telemachos E. Hatziafratis, Integral representation formulas on analytic

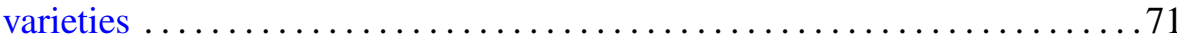

Douglas Austin Hensley, Dirichlet's theorem for the ring of polynomials

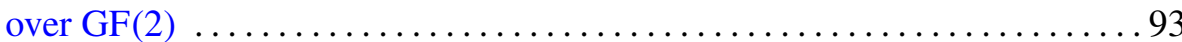

Sofia Kalpazidou, On a problem of Gauss-Kuzmin type for continued

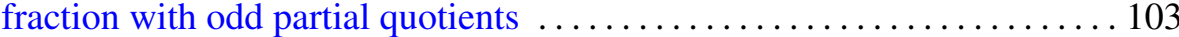

Harvey Bayard Keynes and Mahesh Nerurkar, Ergodicity in affine

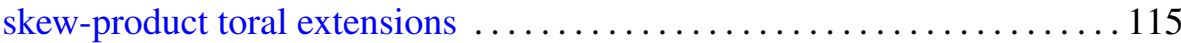

Thomas Landes, Normal structure and the sum-property $\ldots \ldots \ldots \ldots \ldots 127$

Anthony To-Ming Lau and Viktor Losert, Weak*-closed complemented invariant subspaces of $L_{\infty}(G)$ and amenable locally compact groups ...149 Andrew Lelek, Continua of constant distances in span theory . . . . . . . . 161 Dominikus Noll, Sums and products of $B_{r}$ spaces $\ldots \ldots \ldots \ldots \ldots \ldots \ldots \ldots$ Lucimar Nova, Fixed point theorems for some discontinuous operators 189

A. A. S. Perera and Donald Rayl Wilken, On extreme points and support points of the family of starlike functions of order $\alpha$

Massimo A. Picardello, Positive definite functions and $L^{p}$ convolution operators on amalgams ........................

Friedrich Roesler, Squarefree integers in nonlinear sequences ......... 223

Theodore Shifrin, The osculatory behavior of surfaces in $\mathbf{P}^{5}$ 